\title{
La dirección del trabajo independiente mediante el ambiente de enseñanza-aprendizaje adaptativo APA-Prolog
}

\author{
Independent Work Management Through Adaptive Teaching-Learning Environment APA-Prolog
}

A direção do trabalho independente através de ambientes adaptativos de ensino-aprendizagem

Lydia Rosa Ríos-Rodríguez

Universidad de Sancti Spíritus

Sancti Spíritus, Cuba

lidia@uniss.edu.cu

(D) https://orcid.org/0000-0001-5176-931X

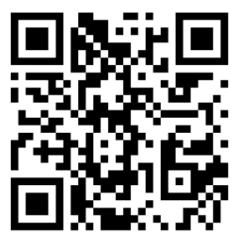

Eldis Román-Cao

Universidad Técnica de Manabí

Portoviejo, Ecuador

eldis.roman@utm.edu.ec

iD https://orcid.org/0000-0002-8552-7906

Yunia Tania Pérez-Medinilla

Universidad de Sancti Spíritus

Sancti Spíritus, Cuba

yunia@uniss.edu.cu

https://orcid.org/0000-0002-9375-9328

Recibido • Received • Recebido: 14 / 03 / 2019

Corregido • Revised • Revisado: 30 / 09 / 2020

Aceptado • Accepted • Aprovado: $26 / 11 / 2020$

\begin{abstract}
Resumen: Alcanzar independencia en el aprendizaje es esencial en la formación profesional. El propósito del artículo es describir el uso del ambiente APA-Prolog en el proceso de enseñanza y aprendizaje ajustado a la dinámica del proceso de dirección del trabajo independiente. El estudio se realizó con un enfoque metodológico cuantitativo, con diseño no experimental de tipo descriptivo. Se aplicó una encuesta, con escala tipo Likert a 22 estudiantes de la carrera Ingeniería Informática de la Universidad de Sancti Spíritus, midió la usabilidad del ambiente una vez ajustado a las fases de planificación, orientación, ejecución y control-evaluación. Los datos se analizaron mediante el cálculo de los valores medios de las respuestas, el por ciento de representatividad y la desviación típica. Los resultados muestran el cumplimiento ordenado de las fases, lo que permitió el reconocimiento de las habilidades de independencia cognoscitiva, la interacción con simuladores, entrenadores, tutoriales, evaluadores, y mapas conceptuales hipermediales, que fueron controlados por agentes inteligentes que tomaron decisiones basadas en los conocimientos previos del estudiantado. Se concluye que se estimuló el desarrollo de habilidades de independencia cognoscitiva, entre ellos lectura, análisis, interpretación, deducción, procesamiento; que el estudiantado gestione el conocimiento mediante la navegación libre o asistida según su elección y el tipo de actividad a resolver, así como que los
\end{abstract}


http://doi.org/10.15359/ree.25-1.11

http://www.una.ac.cr/educare

educare@una.ac.cr

ambientes de enseñanza-aprendizaje adaptativos favorecen un proceso educativo dinámico y centrado en el estudiantado.

Palabras claves: Trabajo independiente; enseñanza asistida por computadora; sistemas adaptativos; ambiente virtual; sistema multiagente.

Abstract: Reaching independence in learning is essential in professional formation. In this line, this article aims to describe the use of the APA-Prolog environment in the teaching-learning process adjusted to the dynamics of the independent work management process. The study was conducted with a quantitative methodological approach and a non-experimental descriptive design. A survey with a Likert scale type was conducted to 22 students from the Computing Engineering program at the University of Sancti Spiritus to measure the use of the environment adjusted to the planning, orientation, execution, and control-evaluation phases. The data were analyzed through the calculus of the mean values of answers, the percentage of representativeness, and the typical deviation. The results showed the ordered fulfillment of the phases that allowed the recognition of the cognitive independence abilities and the interaction with simulators, trainers, tutorials, evaluators, and hypermedia conceptual maps, which were controlled by intelligent agents that made decisions based on the previous students' knowledge. It was concluded that the development of cognitive independence abilities - such as reading, analysis, interpretation, deduction, and processing - was stimulated. It was also concluded that the students manage the knowledge by means of free or assisted browsing according to their choice and the type of activity to solve and that the teachinglearning adaptive environments favor a dynamic educative process based on the student.

Keywords: independent work; computer-assisted teaching; adaptive systems; virtual environment; multi-agent system.

Resumo: Alcançar a independência na aprendizagem é essencial na formação profissional. Esse artigo tem como propósito descrever a utilização do ambiente APA-Prolog no processo de ensinoaprendizagem ajustado à dinâmica do processo de direção do trabalho independente. $O$ estudo foi realizado com um enfoque metodológico quantitativo, e desenho não experimental do tipo descritivo. Foi aplicado um questionário tipo Likert a 22 estudantes da carreira de Engenharia da Computação da Universidade de Sancti Spíritus, medindo o uso do ambiente quando o mesmo foi ajustado as fases de planejamento, orientação, execução e controle-avaliação. A análise dos dados foi feita a partir do cálculo dos valores médios das respostas, a porcentagem percentual e meio ponto percentual. Os resultados demonstram que as fases foram cumpridas ordenadamente, permitindo o reconhecimento de habilidades de independência cognoscitiva, o interagir com simuladores, treinadores, tutoriais, avaliadores e mapas conceituais hiper meios, controlados por agentes inteligentes que fizeram decisões baseadas nos conhecimentos prévios dos estudantes. Conclui-se que foi estimulado o desenvolvimento de habilidades de independência cognoscitiva, entre eles a leitura, análises, interpretação, dedução, processamento de forma que os estudantes gerenciam o conhecimento através da navegação livre ou assistida de acordo com sua escolha e o tipo de atividade a ser resolvida, bem como que os ambientes adaptativos de ensino-aprendizagem são uma alternativa para desenvolver este tipo de trabalho, ao mesmo tempo em que permitem um processo educacional dinâmico e centrado no estudante.

Palavras-chave:Trabalho independente; ambiente assistido por computador; ensino-aprendizagem; sistemas adaptativos; ambiente virtual; sistema multi-agente. 
http://doi.org/10.15359/ree.25-1.11

http://www.una.ac.cr/educare educare@una.ac.cr

\section{Introducción}

El personal docente, quien hasta el presente ha sido el protagonista principal de la universidad, está llamado a convertirse en un facilitador y traspasar dicha preponderancia al estudiantado. Además de ser reconocido como grupo experto en los contenidos que enseña, lo debe ser también en las orientaciones que da a sus estudiantes sobre las formas de estudio a aplicar tanto en la clase como fuera de ella (Díaz Mujica et al., 2017). El profesorado universitario deberá enseñar más que contenidos, las herramientas adecuadas para el aprendizaje por sí mismo.

En este principio basan hoy su trabajo las universidades, empeñadas en egresar profesionales a la altura de su tiempo, capaces de lidiar con la información y resolver problemas de la práctica profesional con alto grado de independencia. En este propósito, es preciso modernizar la forma, los métodos y medios implicados en su aprendizaje. Articular el proceso educativo, los currículos, los contenidos, las competencias y las habilidades de trabajo independiente a las tecnologías es una demanda creciente.

Según Manso Guerra et al. (2015), las tecnologías de la información y las comunicaciones (TIC) poseen una gran influencia en la educación, han creado herramientas y plataformas educativas que apoyan y facilitan el seguimiento del proceso enseñanza-aprendizaje entre las que mencionan: Content Management Systems (CMS), Learning Management Systems (LMS), Learning Content Management Systems (LCMS), repositorios de recursos educativos, herramientas de autor, entre otras.

(Siemens y Mateos, 2010) argumentan que: servicios de información como Google Search, Google Scholar, los dispositivos habilitados con GPS, los libros electrónicos, las publicaciones periódicas en línea o los recursos educativos de acceso libre están mejorando el acceso y la comunicación para estudiantes. Se considera necesario la articulación de los currículos y las actividades de aprendizaje a lo que sucede en estos espacios de información.

De acuerdo con Basso-Aránguiz et al. (2018), las TIC han promovido el cambio de rol de los sujetos protagonistas del proceso educativo y han permitido implementar nuevas formas de interacción sincrónica y asincrónica, al ampliar los espacios y tiempos de aprendizaje, así como las vías para aprender. Por ello asevera que la utilización de las tecnologías representa una oportunidad para realizar innovación educativa en las instituciones de educación superior.

Del mismo modo, gran cantidad de estudios (Cabero Almenara et al., 2017; Duque Méndez, 2009; González et al., 2008; Guerra et al., 2011; Iglesias Rodríguez et al., 2014; Roig-Vila, 2016) aprecian en las tecnologías oportunidades para elaborar nuevos recursos; en ocasiones de carácter general (Almirón y Porro, 2014; Barragán Sánchez, 2005; García Doval, 2005; Ríos Rodríguez et al., 2016; Silva Rodríguez et al., 2009); en modelos a la medida de cada estudiante (Calvi, 1997; Weber y Brusilovsky, 2001) o proponen modelos adaptativos (Lagunes-Domínguez et al., 2017) y apuestan por el aprendizaje móvil. 
http://doi.org/10.15359/ree.25-1.11

http://www.una.ac.cr/educare

educare@una.ac.cr

En relación a la articulación entre las TIC y la oportunidad para mejorar el trabajo independiente del alumnado, Gallardo Echenique et al. (2014) aseguran, a partir de los resultados de una investigación realizada en la Facultad de Ciencias de la Educación y Psicología de la Universidad Rovira i Virgili (URV), que estudiantes con estos recursos prefieren aprender por sí, trabajar de forma independiente, con ayudas de otras personas y estudiar en casa.

El aprendizaje con alto grado de participación del alumnado y mediado por las tecnologías ha tomado posición como uno de los principales esquemas al que aspira la educación; su concepción "ha cambiado los procesos tradicionales de enseñanza y aprendizaje, y proporcionado numerosas ventajas de carácter pedagógico y de acceso" (Tabares et al., 2017, p. 34). Tanto docentes como estudiantes deberán alcanzar cada vez más herramientas didácticas para su implementación, pues estas ofrecen gran potencial para innovar (Rizov y Rizova, 2015). La autonomía es objetivo declarado a promover por la mayoría de los sistemas educativos (Correa-Pérez y Sanhueza-Jara, 2019).

El término trabajo independiente es relacionado también con el de trabajo autónomo y aprendizaje autónomo; sin embargo, poseen aspectos que los diferencian. El primero y segundo refieren a la actividad que realiza cada estudiante en clase o fuera de esta para aprender en relación directa con el profesorado, a partir de su guía, orientación y evaluación (Espinoza-Freire et al., 2017; Pidkasisty, 1986; Román-Cao et al., 2017); el tercero refiere a las estrategias y vías propias empleadas para aprender (Lobato Fraile, 2006; Pegalajar, 2020; Pozo y Monereo, 1999; Uribe Meneses, 2012; Vermunt, 1995).

Se considera que articular un proceso de enseñanza-aprendizaje a la medida de cada aprendiz requiere de un alto grado de preparación de quien enseña. El trabajo independiente, más que una actividad, guía didáctica o momento de trabajo por sí solo, es un proceso complejo organizado por el personal docente antes y durante la clase, organizado y ajustado por el alumnado a su propio ritmo y que concluye una vez comunicado lo aprendido en construcción individual o con ayudas (Román-Cao et al., 2017). Esta dinámica de enseñanza y aprendizaje es definida por Román-Cao y Herrera-Rodríguez (2010) como proceso de dirección del trabajo independiente (PDTI) y es la base teórica de esta investigación.

Resulta novedosa la articulación del PDTI con recursos tecnológicos de aprendizaje como es el caso de la enseñanza asistida por computadora, pues se logra mayor responsabilidad en el proceso de aprendizaje, en la medida en que el alumnado es más autónomo (Correa-Pérez y Sanhueza-Jara, 2019). En el contexto de la educación virtual, "la adaptación hace referencia a los métodos de suministrar personalización en los sistemas de enseñanza-aprendizaje mediados por computador" (González et al., 2008, p. 200).

APA-Prolog es un ambiente de enseñanza-aprendizaje adaptativo que se elaboró con el objetivo de contribuir a mejorar el proceso de enseñanza-aprendizaje de la programación 
http://doi.org/10.15359/ree.25-1.11

descriptiva. Después de dos cursos consecutivos de implementación, se inició, con el apoyo del Equipo de Desarrollo Informático de la Universidad de Sancti Spíritus "José Martí Pérez" (Uniss), la fase de mantenimiento prevista en la metodología de desarrollo de software empleada para su elaboración.

Entre los aspectos a tener en cuenta para un mantenimiento exitoso, se encuentra la opinión del público usuario, en este caso docentes y estudiantes. De modo que se originaron varios problemas de investigación, entre ellos el que se pretende resolver en este trabajo: ¿Qué opinión tendrán estudiantes sobre la pertinencia y usabilidad de APA-Prolog, como herramienta de apoyo al trabajo independiente? Ante esta disyuntiva se determinó que se trabajaría con el objetivo de describir cómo se comporta el uso de APA-Prolog en un proceso de enseñanza y aprendizaje implementado con la dinámica del PDTI.

\section{Metodología}

El estudio se desarrolló desde un enfoque metodológico cuantitativo, con un diseño no experimental de tipo descriptivo. La información se obtuvo a través de una encuesta, con escala tipo Likert, que midió la efectividad de APA-Prolog desde la dinámica del PDTI; su aplicación fue una vez terminada la asignatura Programación Descriptiva en el curso escolar 2016-2017. Posterior a ello se procesó la información por estadística descriptiva y la técnica de cálculo porcentual.

\section{Participantes}

Para la realización del estudio se tomó como población a 43 estudiantes que cursaban el $4^{\text {to }}$ año de la carrera Ingeniería Informática en la Uniss durante los cursos 2015-2016 y 2016-2017, pues fueron quienes interactuaron directamente con APA-Prolog. De este grupo, integraron la muestra de manera intencional 22 estudiantes del curso 2016-2017, que recibían la asignatura en el momento en que se decide medir los indicadores. Participaron 20 del sexo masculino y 2 del femenino, con un promedio de edades de 20 años.

\section{Instrumentos}

Los datos y opiniones de estudiantes se midieron a través de la interacción con APA-Prolog y un cuestionario elaborado sobre la base teórica del PDTI.

\section{APA-Prolog}

APA-Prolog es un ambiente de enseñanza-aprendizaje adaptativo desarrollado por un grupo de docentes del Departamento de Ingeniería Informática de la Universidad de Sancti 
http://doi.org/10.15359/ree.25-1.11

http://www.una.ac.cr/educare

educare@una.ac.cr

Spíritus "José Martí Pérez" cuyos objetivos son: tomar en cuenta los conocimientos previos del estudiantado y apoyar el proceso de enseñanza-aprendizaje de la programación Lógica (Ríos, 2009). La Figura 1 muestra los elementos generales que lo componen.

Figura 1: Componentes de APA-Prolog

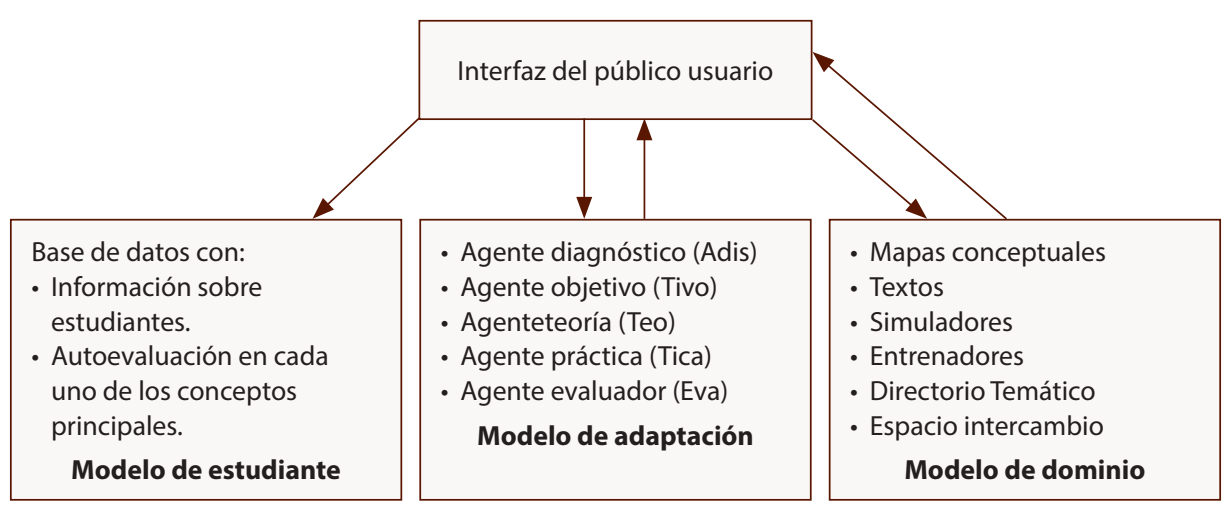

Nota: Elaboración propia con el objetivo de mostrar la dinámica y componentes para apoyar el proceso de enseñanza-aprendizaje de la programación Lógica.

La interfaz de APA-Prolog la constituye un conjunto de mapas conceptuales relacionados entre sí, sobre los cuales navega el estudiantado, que encuentra en su travesía disímiles recursos informáticos entre los que se hallan textos explicativos, entrenadores, tutoriales, simuladores y evaluadores. Al mismo tiempo, un conjunto de agentes inteligentes decide qué recursos activar en cada caso, con base en el estado del conocimiento de cada estudiante (bien, regular o mal).

Para lograr la adaptación, los agentes inteligentes realizan las siguientes tareas:

\section{Agente Adis}

1. Se apropia del nombre y la palabra clave que usará cada estudiante para autentificarse.

2. Captura el grado de conocimiento que posee cada estudiante sobre cada uno de los conceptos fundamentales del ambiente y los recuerda.

3. Investiga si el estudiantado tiene algún objetivo específico que vencer, por ejemplo: los hechos, las reglas, las preguntas, etc.

4. Almacena esos objetivos específicos.

5. Facilita a quien aprende los elementos necesarios para autoevaluarse. 
http://doi.org/10.15359/ree.25-1.11

\section{Agente Tivo}

1. Propone una lista, ordenada por relevancia, de todos los materiales disponibles en APA-Prolog que pudiera ayudar a las necesidades cognitivas del estudiantado.

2. Muestra la secuencia de pasos para lograr el objetivo actual, sugiriendo los enlaces más importantes a seguir, por ejemplo, si el objetivo son los predicados, el camino indicado puede ser: Prolog $\rightarrow$ Programas $\rightarrow$ Predicado.

3. Alerta acerca de los conceptos que son precedentes al concepto objetivo, por ejemplo, si el objetivo fuera predicado, los prerrequisitos serían los conocimientos relacionados con las cláusulas, el functor y el arity de las cláusulas, entre otros.

\section{Agente Teo}

1. Usando las técnicas de mapas adaptativos, y ocultamiento adaptativo de enlaces determina qué variante de mapas mostrar, adiciona o quita enlaces para mostrar una configuración adaptada a su nivel.

2. Decide qué textos presentar en cada concepto, implementando para eso las siguientes técnicas:

a) Variantes de páginas: El sistema almacena dos o tres versiones de una misma página, estas se diferencian en la cantidad de información que contienen y en la profundidad con que es tratada.

b) Explicación de variantes: El contenido tiene diferentes versiones de explicaciones que se muestran de acuerdo con el estado de conocimiento del estudiantado.

c) Coloca en orden descendente de relevancia los enlaces a la información más adecuada según los conocimientos previos del estudiantado.

3. Determina los ejemplos a visualizar, valiéndose de las técnicas anteriores.

4. Suministra una lista ordenada de bibliografía recomendada.

5. Sugiere el orden del próximo concepto a visitar desde el nodo actual.

6. Inserta definiciones de todos los conceptos presentes entre los prerrequisitos para cada nodo.

7. Recomienda ejercicios para entrenamiento y en ocasiones el uso de entrenadores.

8. Recomienda ver simuladores.

9. Recomienda evaluaciones.

10. Motiva para el próximo concepto a visitar. 
http://doi.org/10.15359/ree.25-1.11

http://www.una.ac.cr/educare

educare@una.ac.cr

\section{Agente Tica}

1. Presenta los ejercicios a resolver y las guías para su solución.

2. Ofrece enlaces a prerrequisitos.

3. Refuerza las respuestas correctas.

4. No penaliza las respuestas incorrectas, sino que proporciona elementos importantes a tener en cuenta para llegar a la solución correcta (explicaciones adicionales, explicaciones de prerrequisitos, explicaciones de variantes y explicaciones comparativas).

5. Si el estudiantado no llega a la solución correcta, entonces recomienda estudiar la teoría necesaria para conseguirlo (explicaciones adicionales y variante de páginas).

6. Si el estudiantado responde acertadamente el cuestionario se le recomiendan documentos y materiales para profundizar.

7. Recomienda pasar al evaluador.

Los recursos fundamentales que Tica manipula son los entrenadores.

\section{Agente Eva}

1. Selecciona el cuestionario teniendo en cuenta el concepto y el estado de conocimiento del estudiantado en ese concepto.

2. Evalúa cualitativamente.

3. Recomienda pasar o no al siguiente concepto en el mapa.

4. Sugiere otros recursos para apropiarse del contenido cuando el estudiantado no es capaz de responder correctamente alguna pregunta del examen.

5. Orienta bibliografía para profundizar si el estudiantado ha respondido correctamente.

6. No penaliza al estudiantado, si los resultados no son los esperados, sino que hace recomendaciones para superar las dificultades encontradas.

7. Si el estudiantado responde con éxito el formulario que le corresponde, pasa su estado de conocimiento a un nivel superior (bien o regular).

8. Si el estudiantado no responde con éxito el formulario que le corresponde, entonces pasa su estado de conocimiento a un nivel inferior (regular o mal). 
http://doi.org/10.15359/ree.25-1.11

http://www.una.ac.cr/educare educare@una.ac.cr

En la Figura 2 se muestra, la responsabilidad de cada uno de los agentes del sistema y la interacción entre los diferentes componentes que lo integran durante la navegación asistida.

Figura 2: Interacción de los componentes durante la navegación asistida

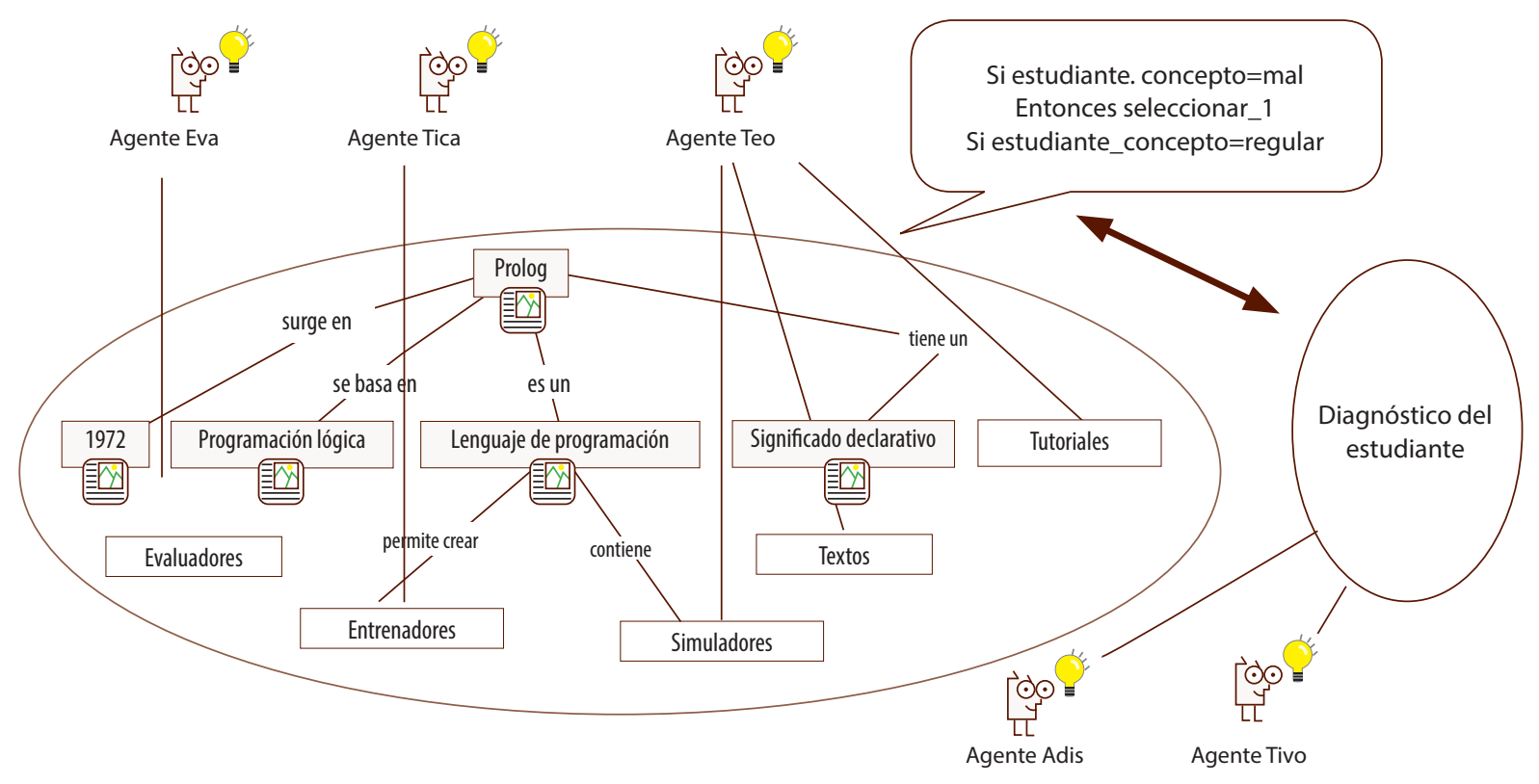

Nota: La figura es de elaboración propia y tiene como objetivo mostrar la responsabilidad de cada uno de los agentes del sistema y la interacción entre los diferentes componentes que lo integran durante la navegación asistida.

\section{Procedimiento}

APA-Prolog fue ajustado a las cuatro fases (momentos) para la dirección del trabajo independiente, estas fases coinciden con las propuestas por Román-Cao y Herrera-Rodríguez (2010).

Fase 1: Planificación y determinación del tipo de trabajo independiente.

Fase 2: Orientación.

Fase 3: Ejecución o participación activa del estudiantado en el trabajo independiente.

Fase 4: Control y evaluación.

Se presentan a continuación las principales actividades que se realizaron siguiendo la dinámica que se resume en la Figura 3. 
http://doi.org/10.15359/ree.25-1.11

http://www.una.ac.cr/educare

educare@una.ac.cr

Figura 3: El trabajo independiente con el empleo de APA-Prolog

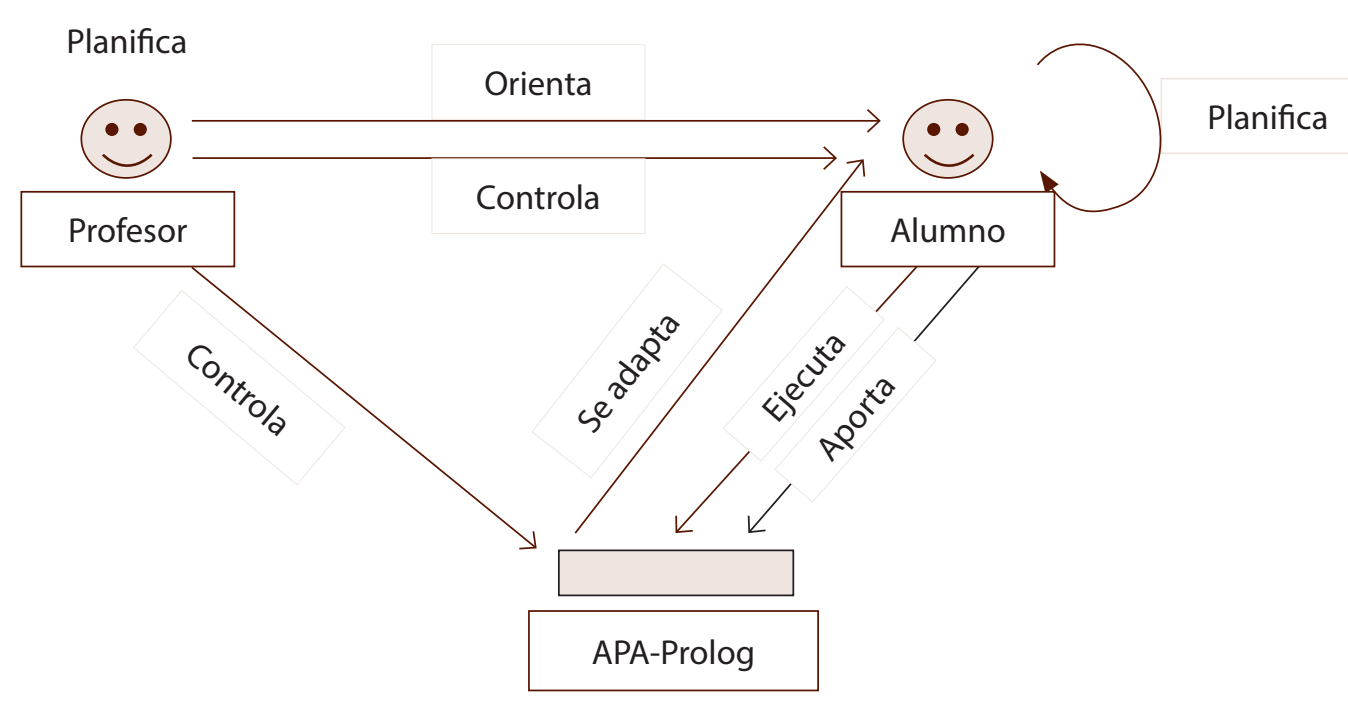

Nota: La figura es de elaboración propia y tiene como objetivo mostrar la responsabilidad de cada participante del trabajo independiente en su interacción con APA-Prolog.

Fase 1: Planificación y determinación del tipo del trabajo independiente.

Durante este primer momento el profesorado realizó las siguientes actividades:

1. Dividió el contenido a enseñar en temas o unidades lógicas de contenido.

2. Determinó qué recursos del ambiente el estudiantado podía utilizar en cada caso. Por ejemplo, en la actividad orientada al estudio del proceso de matching en Prolog, el estudiantado interactuó con el simulador de matching que forma parte del ambiente, en las actividades orientadas al backtracking pudieron proceder de igual manera, pero con el simulador de backtracking y así sucesivamente.

¿Por qué usar los simuladores en estos casos? Porque estos son procesos que no resultan transparentes para el equipo programador, sin embargo, deben conocerlos en profundidad para poder utilizarlos en la programación durante su vida profesional. Del mismo modo orientó la navegación por el ambiente hacia tutoriales, si lo importante a revisar eran elementos teóricos, o hacia entrenadores, si se trataba de actividades prácticas a realizar o hacia evaluadores si se requería evaluar.

3. Determinó qué conceptos el estudiantado podía visitar en el ambiente en cada caso específico. 
http://doi.org/10.15359/ree.25-1.11

4. Determinó qué nuevos recursos para el ambiente el estudiantado podía confeccionar como parte del trabajo independiente.

5. Determinó los valores humanos posibles a potenciar o trabajar en alguna medida. Ellos fueron: laboriosidad, responsabilidad, patriotismo y humanismo.

Fase 2: Orientación.

En esta fase el profesorado condujo a sus estudiantes hacia lo que necesitaban conocer, indicándoles:

a) cuáles son los contenidos a estudiar,

b) cuáles son las habilidades a alcanzar,

c) qué conceptos del mapa se revisarían,

d) qué recursos deben utilizar,

e) qué aportará derivado de la realización de las actividades orientadas: (una nueva base de conocimiento, mejoras a una ya existente o un nuevo recurso para el ambiente),

f) cómo y cuándo evaluará lo aprendido.

Fase 3: Ejecución o participación activa del estudiantado en el trabajo independiente.

En este momento, estudiantes realizaron las siguientes acciones:

1. Interactuaron con el ambiente y se autoevaluaron.

2. En dependencia de la autoevaluación navegaron por recursos seleccionados por APAProlog.

3. Si creyeron necesario, se sometieron a ser evaluados por el ambiente.

4. Si fueron evaluados de bien, recibieron recomendaciones para reafirmar lo aprendido.

5. Continuaron navegando y consultando recursos hasta ser evaluados de bien en el tema que estudiaron.

Por su parte APA-Prolog tomó las siguientes decisiones:

1. Si el estudiantado se autoevaluó o evaluó de mal por el ambiente, este se adaptó, mostró mapas y recursos adecuados, recomendó bibliografía y otros conceptos a visitar para asegurar los conocimientos que era preciso tener para apropiarse del contenido objeto de estudio, además advirtió al estudiantado para qué le serviría este nuevo concepto. 
http://doi.org/10.15359/ree.25-1.11

http://www.una.ac.cr/educare

educare@una.ac.cr

2. Si se autoevaluó o evaluó por el ambiente de regular, este se adaptó y mostró mapas y recursos adecuados.

3. Si se autoevaluó o evaluó por el ambiente de bien, este brindó recursos para profundizar en nuevos contenidos de la asignatura.

Durante este momento resultó determinante el papel de los agentes inteligentes en la adaptación y selección de los recursos a la medida de cada estudiante.

Fase 4: Control y evaluación.

En esta fase el profesorado:

1. Realizó la evaluación participativa y auxiliándose del propio ambiente, así como, de las respuestas dadas por el estudiantado a los cuestionarios de entrenadores y evaluadores.

2. Premió los mejores trabajos colocándolos en el ambiente como nuevos recursos para ser visitados y valorados por el resto de estudiantes, explotando la evaluación como un elemento de diagnóstico y formación.

3. Se retroalimentó de los resultados presentados por sus estudiantes.

4. Motivó para profundizar en temas que provocaron dudas en sus estudiantes.

5. Actualizó el diagnóstico del estudiantado lo que hizo posible reestructurar las acciones que permitieran su avance hasta niveles de desarrollo superiores.

Mientras, el estudiantado pudo:

1. Socializar la información que emanó de su propio esfuerzo.

2. Retroalimentarse por vía electrónica, mediante correos, foros o chat, también disponibles en el ambiente.

3. Participar en el debate propiciado por su docente en el aula en algún momento del proceso, tomando como base alguno de los recursos disponibles en el ambiente.

\section{Cuestionario para recoger las opiniones de estudiantes sobre el empleo de APA- Prolog en el trabajo independiente}

La encuesta se elaboró a partir de los indicadores propuestos por (Román, 2013). Se organizó tomando en cuenta la medición, primero, del logro de habilidades propias para trabajar de manera independiente, refiriendo 7 aspectos: nivel de desarrollo alcanzado por el estudiantado; orientación adecuada de las actividades; medios y recursos de aprendizaje empleados; participación activa en las evaluaciones y autoevaluaciones; posibilidad de comunicar o publicar los resultados alcanzados y aplicabilidad en otras asignaturas. Luego se 
http://doi.org/10.15359/ree.25-1.11

midió, derivado de las anteriores, el desarrollo de la independencia cognoscitiva del alumnado, particularmente la estimulación de la lectura, el análisis, la deducción, el procesamiento de información y la capacidad para llegar a conclusiones.

Para la medición de las distintas variables, relacionadas con las habilidades de trabajo independiente, particularmente en las preguntas 1, 2, 3, 5, 6 y 7 se utilizó una escala Likert con una puntuación de 5 a 1, reportada como válida por García Sánchez et al. (2011). Esta comprende desde las opciones totalmente de acuerdo hasta en total desacuerdo, y la posición intermedia es indiferente. Para examinar la actitud de la persona encuestada se calculó el promedio de los valores asignados a cada pregunta. Para conocer la dispersión de los datos alrededor de la media se calculó la desviación típica.

Antes de la aplicación del cuestionario se informó a los sujetos participantes sobre los propósitos del estudio, así como de la importancia de su participación consciente para lograr de APA-Prolog un mejor producto y una herramienta que apoye adecuadamente el proceso de enseñanza-aprendizaje del estilo descriptivo de programación. Durante los 20 minutos aproximados que duró su aplicación, se proporcionaron los materiales necesarios y las asistencias para la aclaración de términos propios del lenguaje didáctico e informático.

\section{Resultados de la encuesta}

Los valores medios de las respuestas a las interrogantes 1,2,3,5,6 y 7 de la encuesta aplicada se muestran en la Tabla 1:

Tabla 1: Opiniones sobre el empleo de APA-Prolog para el trabajo independiente

\begin{tabular}{|c|c|c|c|c|c|c|c|c|}
\hline & Enunciados de los aspectos a medir & M & DT & $\%$ & $\%$ & $\begin{array}{c}\% \\
1\end{array}$ & $\begin{array}{l}\% \\
\mathrm{D}\end{array}$ & $\begin{array}{l}\% \\
\text { TD }\end{array}$ \\
\hline 1 & $\begin{array}{l}\text { Las actividades para trabajo independiente que se orientaron en el } \\
\text { transcurso de la asignatura estuvieron en dependencia del nivel de } \\
\text { desarrollo alcanzado por usted. }\end{array}$ & 4,7 & 0,6 & 81,8 & 9,09 & 9,09 & 0 & 0 \\
\hline 2 & Las actividades a realizar siempre estuvieron claramente orientadas. & 4,5 & 0,9 & 68,2 & 13,6 & 13,6 & 4,5 & 0 \\
\hline 3 & $\begin{array}{l}\text { Los medios disponibles en APA-Prolog para la realización del trabajo } \\
\text { independiente resultaron adecuados. }\end{array}$ & 4,9 & 0,6 & 95,5 & 0 & 0 & 4,5 & 0 \\
\hline 5 & $\begin{array}{l}\text { APA-Prolog le permitió participar de manera activa en la evaluación de } \\
\text { su propio aprendizaje. }\end{array}$ & 4,5 & 0,7 & 63,6 & 22,7 & 13,6 & 0 & 0 \\
\hline 6 & La posibilidad que ofrece APA-Prolog para publicar sus resultados es buena. & 3.8 & 0,9 & 22,7 & 45,5 & 22,7 & 9,1 & 0 \\
\hline 7 & $\begin{array}{l}\text { Sistemas similares a APA-Prolog se podrían utilizar en otras asignaturas } \\
\text { para realizar el trabajo independiente. }\end{array}$ & 4,7 & 0,7 & 86,4 & 4,5 & 4,5 & 4,5 & 0 \\
\hline
\end{tabular}

Leyenda: M: Media, DT: Desviación típica, TA: Totalmente de acuerdo, A: De acuerdo, I: Ni de acuerdo ni en desacuerdo (indiferente), D: En desacuerdo, TD: Totalmente en desacuerdo.

Nota: Elaboración propia. 
http://doi.org/10.15359/ree.25-1.11

http://www.una.ac.cr/educare

educare@una.ac.cr

La primera pregunta está relacionada con las posibilidades de los sistemas adaptativos, en cuanto a adecuarse a las características del estudiantado que interactúa con él. En este sentido, la navegación asistida estuvo respaldada por varios agentes inteligentes que tomaron decisiones a partir en los conocimientos previos del estudiantado y seleccionaron los recursos adecuados en cada caso. El valor medio de las respuestas igual a 4,7 demuestra que reconocen esta posibilidad de APA-Prolog.

A la segunda interrogante, relacionada con el momento de orientación que desarrolló el profesorado, tres estudiantes respondieron que no estaban de acuerdo ni en desacuerdo, es decir, tomaron una posición indiferente y uno marcó la casilla en desacuerdo, opiniones que influyeron en la obtención de 4,5 como valor medio, lo que constituye una alerta para el personal docente, quien debe replantearse sus actividades para cursos posteriores.

El valor medio igual a 4,9 en las respuestas a la tercera pregunta ilustra la aceptación de los variados medios disponibles en APA-Prolog, los que permitieron al estudiantado adquirir habilidades en el manejo efectivo de los simuladores, entrenadores, tutoriales, evaluadores y mapas conceptuales hipermediales.

De igual modo pudieron interpretar lo que leyeron, evaluaron alternativas, resumieron y guardaron la información necesaria, estas constituyen otras de las habilidades que reforzaron.

Al valorar si APA-Prolog le permitió participar de manera activa en la evaluación de su propio aprendizaje, la mayoría afirmó estar totalmente de acuerdo, cinco que estaban de acuerdo y tres que ni de acuerdo ni en desacuerdo. En este resultado influyó que los mapas conceptuales que forman parte del ambiente fueron confeccionados por personal experto e incluyen toda la información de un tema, lo que permitió al estudiantado, en todo momento, saber cuánto ha aprendido y cuánto le falta por aprender. La Figura 4 muestra uno de esos mapas conceptuales.

A estos resultados también contribuyeron los evaluadores presentes en el ambiente.

La evaluación desarrollada adquirió nueva dimensión, pues el profesorado pudo premiar los mejores trabajos de curso agregándolos al mapa como nuevos recursos y constituyendo ejemplos a consultar por otros grupos de estudiantes. El hecho de que un trabajo de su autoría está publicado en el ambiente y por tanto en internet, cambia la forma en que el estudiantado comunicaba sus resultados hasta ese momento, concediéndole mayor relevancia. Además, estimula la formación de valores como la laboriosidad y la responsabilidad. Esto contrasta con el valor medio más bajo en las respuestas, el cual se obtuvo en la pregunta 6, más no hubo ninguna posición en total desacuerdo con la posibilidad que ofrece APA-Prolog para publicar los resultados, sino que la mayoría de las selecciones se concentraron entre de acuerdo y en desacuerdo. 
http://doi.org/10.15359/ree.25-1.11

Figura 4: Mapa conceptual que brinda las nociones más generales sobre Prolog

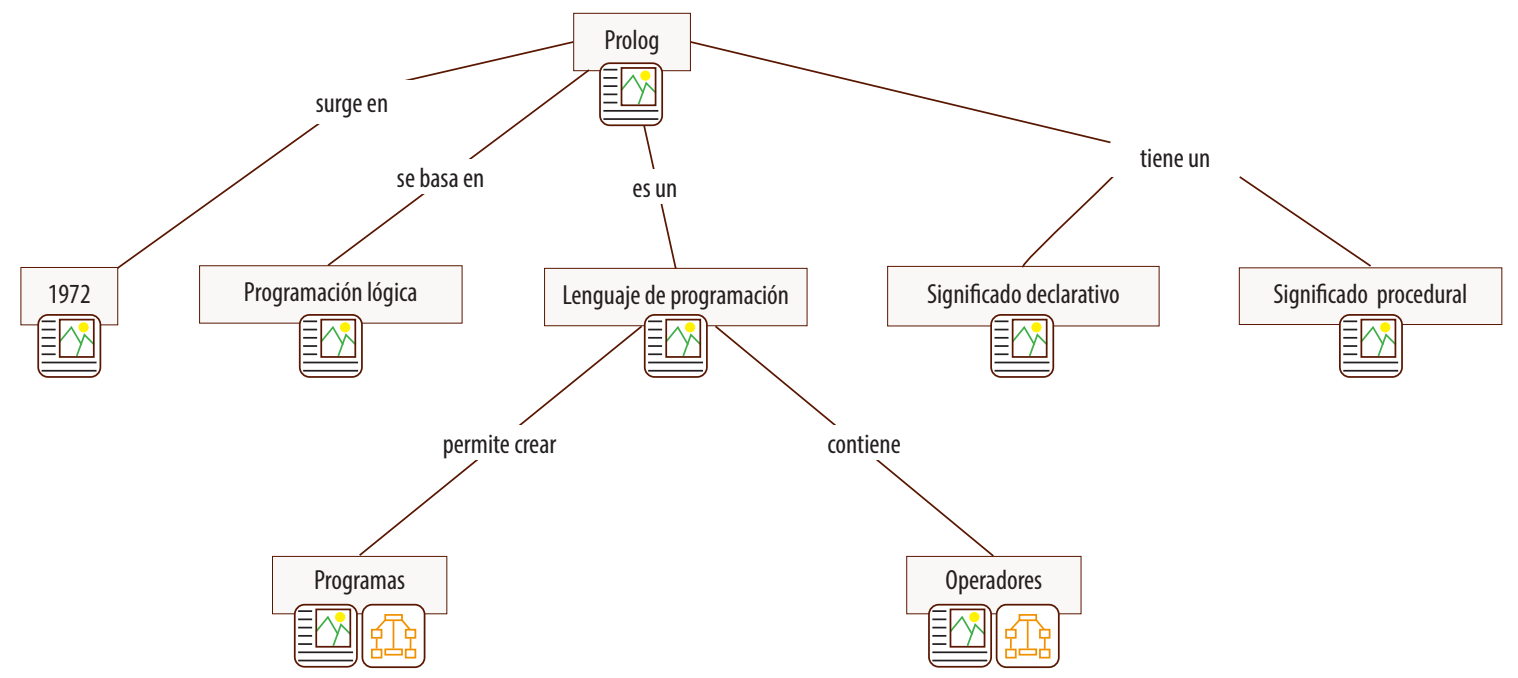

Nota: La Figura es de confección propia y tiene como objetivo mostrar resultados elaborados por los estudiantes en la construcción de mapas conceptuales mediante APA-Prolog.

El ambiente también incluye espacios para foros de discusión y chateo, los que, de igual manera, facilitaron la comunicación y socialización de los resultados alcanzados durante las sesiones de trabajo independiente y donde el estudiantado interactuó para solucionar las actividades orientadas. Una media igual a 4,7 en las repuestas a la pregunta 7 indica que el empleo de APA-Prolog como herramienta para el trabajo independiente dejó en el estudiantado una alta motivación.

En relación con las habilidades de independencia cognoscitiva se midió el reconocimiento que hace el estudiantado sobre la necesidad de desarrollarlas para lograr un adecuado aprendizaje desde el trabajo independiente. Araya Ramírez (2014) las considera como habilidades intelectuales demostradas por los individuos al realizar una tarea por sí solos; esto les permite apropiarse del conocimiento para resolver problemas y transformar su entorno.

Macías Vázquez (2017) las relaciona con las habilidades para pensar (thinking skills), es decir, como ciertas capacidades mentales que permiten a las personas captar, procesar e interpretar información, y que pueden ser enseñadas. Son operaciones lógicas del pensamiento por medio de las cuales el sujeto se apropia de los contenidos y del proceso que usó para ello (FríaskGuzmán et al., 2017). Según Ortiz Gómez (2010), se relacionan con la cognición, es decir, conocer, reconocer, organizar y utilizar el conocimiento.

En la Tabla 2 se muestra el porcentaje de estudiantes que reconoce haber requerido de algunas de estas habilidades para realizar el trabajo independiente y apropiarse de los contenidos disponibles en APA-Prolog. Estos se obtuvieron a partir de las respuestas a la pregunta 4 del cuestionario. 
http://doi.org/10.15359/ree.25-1.11

http://www.una.ac.cr/educare

educare@una.ac.cr

Tabla 2: Reconocimiento de habilidades de independencia cognoscitiva en la interacción con APA-Prolog

\begin{tabular}{lr} 
El alumnado en su interacción con los recursos de APA-Prolog percibió la necesidad de: & $\%$ \\
\hline Leer & 100 \\
Interpretar & 100 \\
Analizar & 100 \\
Deducir & 86,36 \\
Procesar & 86,36 \\
Concluir & 81,81
\end{tabular}

Nota: Elaboración propia.

Lo más significativo de esta medición es mostrar que el 92,4\% de estudiantes reconoce la necesidad de desarrollar habilidades cognitivas como requisito necesario para realizar trabajos autónomos en APA-Prolog, pues en la medida que se lee más y con mayor facilidad, se alcanzarán mejores resultados. La lectura del material le encausará por caminos lógicos del pensamiento como el procesamiento de información, la interpretación, el análisis y la deducción, necesarios para la construcción de conclusiones acertadas.

Este criterio es reforzado en el estudio de Carballo Nápoles y Pérez-Suárez (2011), quienes manifiestan que la independencia cognoscitiva incita la capacidad para pensar y trabajar con originalidad de manera independiente, para lograr vencer, eficientemente, con métodos y estrategias individuales, los inconvenientes que surgen en la realización de actividades de trabajo independiente. Se considera que en la medida en que se logre identificar, utilizar y desarrollar las habilidades cognitivas, se lograrán mejores resultados de aprendizaje de manera autónoma.

Así mismo, en una valoración sobre la actitud de cada persona encuestada hacia el empleo de APA-Prolog desde la dinámica del PDTI resultó que no hubo puntuaciones para desfavorables, el $4,5 \%$ se ubica en el intervalo ni de acuerdo ni en desacuerdo y en desacuerdo, mientras que el $72 \%$ manifiesta una actitud favorable, lo que muestra su aceptación. La Figura 5 facilita la comprensión de este resultado. 
http://doi.org/10.15359/ree.25-1.11

http://www.una.ac.cr/educare educare@una.ac.cr

Figura 5: Resumen de puntuaciones dadas por los encuestados

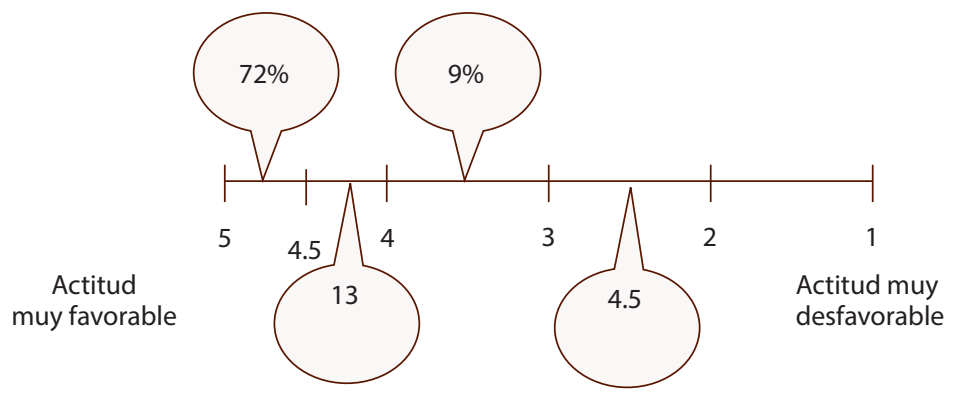

Nota: Elaboración propia, derivada de la aplicación integral de instrumentos.

Este mismo análisis reveló la existencia de un estudiante que no muestra una actitud favorable hacia APA-Prolog desde la dinámica del PDTI, esto se aprecia a través del promedio de las respuestas que otorgó a las interrogantes del cuestionario: 2,3. De igual modo dos estudiantes, con promedios de 3,3 y 3,7 requieren ser atendidos (Figura 6).

Figura 6: Promedios de las respuestas otorgadas por cada estudiante a las interrogantes de la encuesta

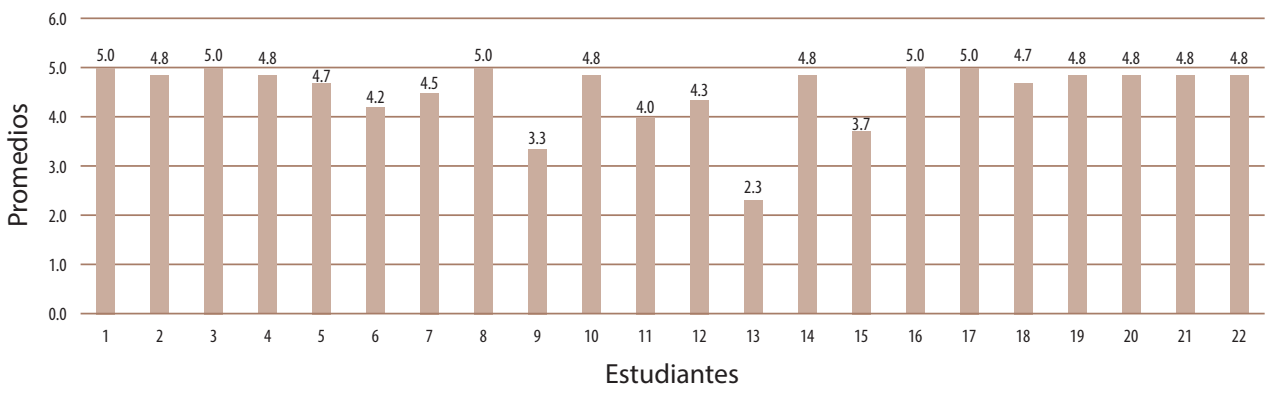

Nota: Elaboración propia, derivada de la aplicación integral de instrumentos.

En términos generales el indicador más valorado por el estudiantado fue el referido a los medios digitales que APA-Prolog facilita para la realización del trabajo independiente $(M=4,9$; DT=0,6), donde el 95,5\% está "totalmente de acuerdo". Le siguen, con iguales valores medios, aquellos en que estiman que APA-Prolog se adapta a sus necesidades $(M=4,7 ; D T=0,6)$ donde el 81,8\% está "totalmente de acuerdo" y el 9,09\% está "de acuerdo" y en el que expresan que les gustaría contar con recursos informáticos similares en otras asignaturas ( $M=4,7 ; D T=0.7)$, en este caso el 86,4\% marcó "totalmente de acuerdo" y el 4,5\% "de acuerdo". La puntuación más baja la obtuvo la posibilidad que ofrece APA-Prolog para publicar los resultados de estudiantes ( $M=3,8$; DT=0,9), el 22,7\% manifestó estar "totalmente de acuerdo" y el 45,5\% "de acuerdo" mientras el $22,7 \%$ adoptó una posición indiferente y el 9,1\% reveló estar en "desacuerdo". 
http://doi.org/10.15359/ree.25-1.11

http://www.una.ac.cr/educare

educare@una.ac.cr

\section{Discusión y conclusiones}

Los resultados del estudio indican que el estudiantado valora positivamente el uso de APAProlog como herramienta de apoyo al PDTI, pues ofrece una dinámica de estudio interactiva y participativa donde la diversidad de medios por los que reciben la información es uno de los principales atractivos. Esto se corresponde con la presencia, hoy, en las aulas universitarias de jóvenes que han crecido en la sociedad de la información, quienes prefieren tomar datos de manera simultánea de diversas fuentes, comunicarse permanentemente, así como haberse desarrollado en un entorno altamente informatizado (Almirón y Porro, 2014).

La articulación de APA-Prolog al PDTI logró que el estudiantado gestionara el conocimiento mediante la navegación libre o asistida en dependencia de su elección y el tipo de actividad a resolver. Este aspecto también recibió una elevada puntuación.

El cumplimiento ordenado de las fases de planeación, orientación, ejecución y evaluación permitió la interacción con simuladores, entrenadores, tutoriales, evaluadores, y mapas conceptuales hipermediales, lo que fue controlado por agentes inteligentes que tomaron decisiones basadas en los conocimientos previos del estudiantado. Estos resultados concuerdan con los obtenidos por Avila y Bosco (2001), quienes, en una investigación sobre los ambientes virtuales de aprendizaje, manifiestan que la práctica educativa les demostró que la incorporación de las tecnologías por sí mismas no tienen significado educativo, se requiere que estas vayan acompañadas de un modelo pedagógico innovador y creativo que le dé sentido a su uso.

Por otro lado, el empleo de APA-Prolog contribuyó al fomento y desarrollo, en el estudiantado, de las habilidades de independencia cognoscitiva: lectura, interpretación y análisis, en mayor medida y las de deducir, procesar y concluir, en menor medida. Estos resultados también coinciden con los obtenidos por Avila y Bosco (2001), quienes aseguran que las tecnologías permiten diferentes formas de lectura no lineales en donde el sujeto va creando y recreando los contenidos en función de sus intereses, aptitudes y actitudes frente al objeto de estudio, lo cual le obliga a desarrollar habilidades del pensamiento distintas a las que posee, pues requiere apropiarse del conocimiento para aplicarlo a su práctica cotidiana.

Asimismo, el ejercicio frecuente de la autoevaluación e interacción con entrenadores y evaluadores propició una participación activa del estudiantado en la medición de su propio aprendizaje, lo cual es reconocido por el 86,3\%.

Resulta novedosa la articulación del PDTI con recursos tecnológicos de aprendizaje adaptativos, pues se logra mayor responsabilidad en el proceso de quien aprende en la medida que se hacen personas más autónomas (Correa-Pérez y Sanhueza-Jara, 2019).

La dirección del trabajo independiente, en su concepción como proceso de dirección, constituye una novedad para el proceso formativo de la Uniss, más aún cuando se utilizan sistemas informáticos que propician un uso eficiente y sistemático de las TIC como es el caso de 
http://doi.org/10.15359/ree.25-1.11

http://www.una.ac.cr/educare educare@una.ac.cr

APA-Prolog. Se considera una alternativa a emplear en la educación superior, pues permite la asignación de nuevos roles a docentes y a estudiantes. Se alza como un mecanismo que ayuda a la persona que aprende para alcanzar un nivel de protagonismo superior. El procesamiento y la generación del conocimiento son responsables principales de la gestión.

De manera general, el aporte fundamental que se hace es el procedimiento que se presenta para la implementación de una enseñanza y aprendizaje centrada en el trabajo independiente mediante el uso del ambiente APA-Prolog y la particularidad metodológica que se sigue con las fases de planificación, orientación, ejecución y control-evaluación. Se recomienda realizar estudios similares teniendo en cuenta el criterio de docentes, la materia, los resultados de la interacción de estudiantes con el ambiente, particularmente con entrenadores y evaluadores, así como, dando atención a las habilidades cognitivas de deducir, procesar y concluir.

\section{Referencias}

Almirón, M. E. y Porro, S. (2014). Los docentes en la sociedad de la información: Reconfiguración de roles y nuevas problemáticas. IE Comunicaciones: Revista Iberoamericana de Informática Educativa, 19, 17-31. https://dialnet.unirioja.es/servlet/revista?codigo=12765

Araya Ramírez, N. (2014). Las habilidades del pensamiento y el aprendizaje significativo en matemática, de escolares de quinto grado en Costa Rica. Revista Actualidades Investigativas en Educación, 14(2), 1-30. http://www.redalyc.org/pdf/447/44731371003.pdf

Avila, P. y Bosco, M. D. (2001). Ambientes virtuales de aprendizaje una nueva experiencia. 20th International Council for Openand Distance Education. Germany. Germany. http:// investigacion.ilce.edu.mx/panel control/doc/c37ambientes.pdf

Barragán Sánchez, R. (2005). El portafolio, metodología de evaluación y aprendizaje de cara al nuevo espacio europeo de educación superior. Una experiencia práctica en la Universidad de Sevilla. Revista Latinoamericana de Tecnología Educativa, 4(1), 121-140. https://relatec. unex.es/article/view/189

Basso-Aránguiz, M., Bravo-Molina, M., Castro-Riquelme, A., y Moraga-Contreras, C. (2018). Propuesta de modelo tecnológico para Flipped Classroom (T-FliC) en educación superior. Revista Electrónica Educare, 22(2), 1-17. https://doi.org/10.15359/ree.22-2.2

Carballo Nápoles, E. Y. y Pérez-Suárez, A. (2011). El impacto social de la independencia cognoscitiva en el modo de actuación del adolescente cubano. Innovación Tecnológica, $17(2), 1-5$.

Cabero Almenara, J., Roig-Vila, R. y Mengual-Andrés, S. (2017). Conocimientos tecnológicos, pedagógicos y disciplinares de los futuros docentes según el modelo TPACK. Digital Education Review, 32, 73-84. https://dialnet.unirioja.es/servlet/articulo?codigo=6295105 
http://doi.org/10.15359/ree.25-1.11

http://www.una.ac.cr/educare

educare@una.ac.cr

Calvi, L. (1997, June). Multifunctional (Hyper) books: A cognitive perspective (or the user's side). En P. Brusilovsky, J. Fink y J. Kay (Eds.), Workshop "Adaptative Systems and User Modeling on the World Wide Web", Sixth Internatiional Conference on User Modeling. Sardinia, Italia. http://www.contrib.andrew.cmu.edu/ plb/UM97_workshop/Calvi.html

Correa-Pérez, R. y Sanhueza-Jara, M. G. (2019). Characterization of autonomous work in a Chilean english pedagogy program: Teacher' and freshmen's perspectives. Revista Electrónica Educare, 23(1), 1-23. https://doi.org/10.15359/ree.23-1.4

Díaz Mujica, A., Pérez Villalobos, M. V., González-Pienda, J. A. y Núñez Pérez, J. C. (2017). Impacto de un entrenamiento en aprendizaje autorregulado en estudiantes universitarios. Perfiles Educativos, 39(157), 87-104. https://doi.org/10.22201/iisue.24486167e.2017.157.58442

Duque Méndez, N. D. (2009). Modelo adaptativo multi-agente para la planificación y ejecución de cursos virtuales personalizados [Tesis doctoral]. Universidad Nacional de Colombia. https:// pdfs.semanticscholar.org/629a/94a0d57b8915766c9909bfdafb90b1308ec9.pdf

Espinoza-Freire, C. E., Serrano Polo, O. y Brito Paredes, P. (2017). El trabajo autónomo en estudiantes de la Universidad Técnica de Machala. Universidad y Sociedad, 9(2), 202-212. https://rus.ucf.edu.cu/index.php/rus/article/view/569

Frías Guzmán, M., Haro Águila, Y. y Artiles Olivera, I. (2017). Las habilidades cognitivas en el profesional de la información desde la perspectiva de proyectos y asociaciones internacionales. Investigación Bibliotecológica, 31(71), 201-218. http://dx.doi.org/10.22201/ iibi.0187358xp.2017.71.57816

Gallardo Echenique, E. E., Marqués Molías, L. y Bullen, M. (2014). Usos académicos y sociales de las tecnologías digitales del estudiante universitario de primer año. Tendencias Pedagógicas, 23, 191-204. https://revistas.uam.es/tendenciaspedagogicas/article/view/2079

García Doval, F. (2005). El papel de los portafolios electrónicos en la enseñanza-aprendizaje de las lenguas. Glosas Didácticas, 14, 112-119. https://www.um.es/glosasdidacticas/GD14/10.pdf

García Sánchez, J., Aguilera Terrats, J. R. y Castillo Rosas, A. (2011). Guía técnica para la construcción de escalas de actitud. Odiseo, Revista Electrónica de Pedagogía, 8(16), 1-13. http://www.odiseo.com.mx/2011/8-16/pdf/garcia-aguilera-castillo-guia-construccionescalas-actitud.pdf

Guerra, A., Giugni, M. y Fernández, J. (2011). Sistema hipermedia adaptativo basado en agentes inteligentes para soporte a la educación semipresencial. Revista de Tecnología de Información y Comunicación en Educación, 5(2), 45-60. https://docplayer.es/15140091Sistema-hipermedia-adaptativo-basado-en-agentes-inteligentes-para-soporte-a-laeducacion-semipresencial.html 
http://doi.org/10.15359/ree.25-1.11

http://www.una.ac.cr/educare educare@una.ac.cr

González, H. M., Duque, N. D. y Ovalle, D. A. (2008). Modelo del estudiante para sistemas adaptativos de educación virtual. Revista Avances en Sistemas e Informática, 5(1), 199-206. http://www.redalyc.org/pdf/1331/133114993004.pdf

Iglesias Rodríguez, A., Olmos Migueláñez, S., Torrecilla Sánchez, E. M. y Mena Marcos, J. J. (2014). Evaluar para optimizar el uso de la plataforma moodle (studium) en el departamento de didáctica, organización y métodos de investigación. Tendencias Pedagógicas, 23, 155-170. https://revistas.uam.es/tendenciaspedagogicas/article/view/2077

Lagunes-Domínguez, A., Torres-Gastelú, C. A., Angulo-Armenta, J. y Martínez-Olea, M. Á. (2017). Prospectiva hacia el aprendizaje móvil en estudiantes universitarios. Formación Universitaria, 10(1), 101-108. https://doi.org/10.4067/S0718-50062017000100011

Lobato Fraile, C. (2006). El estudio y trabajo autónomo del estudiante. En M. de Miguel. (Dir.), Métodos y modalidades de enseñanza centradas en el desarrollo de competencias (pp. 1-30). Ediciones Universidad de Oviedo.

Macías Vázquez, A. (2017). El colapso del capitalismo tecnológico. Escolar y Mayo.

Manso Guerra, Y., Cañizares González, R. y Febles Rodríguez, J. P. (2015). Plataforma educativa ZERA: Modelo de adaptación de contenidos sensible al contexto. Digital Education Review, 27, 154-164. http://revistes.ub.edu/index.php/der/article/view/11606

Ortíz Gómez, G. (2010). Habilidades básicas del pensamiento. Texto basado en el desarrollo de competencias con enfoque en el modelo META. Cengage Learning.

Pegalajar, M. C. (2020). Estrategias de trabajo autónomo en estudiantes universitarios noveles de educación. REICE. Revista Iberoamericana sobre Calidad, Eficacia y Cambio en Educación, 18(3), 29-45. https://doi.org/10.15366/reice2020.18.3.002

Pidkasisty, P. (1986). La actividad cognoscitiva independiente de los alumnos en la enseñanza. Pueblo y Educación.

Pozo, J. I. y Monereo, C. (1999): El aprendizaje estratégico. Santillana.

Ríos, L. (2009). Ambiente de enseñanza-aprendizaje inteligente para la programación lógica [Tesis de Doctorado]. Universidad Central Marta Abreu de Las Villas, Cuba.

Ríos Rodríguez, L. R., Toledo Martín, T. R. y Fuentes Chaviano, P. (2016, febrero). Los ambientes de enseñanza-aprendizaje adaptativos, una alternativa para estudiantes con discapacidad motora. Ponencia presentada en Universidad 2016. X Congreso Internacional de Educación Superior. Universidad de La Habana, Cuba.

Rizov, T. y Rizova, E. (2015). Augmented reality as a teaching tool in higher education. International Journal of Cognitive Research in Science, Engineering and Education (IJCRSEE), 3(1), 7-15. https://www.ijcrsee.com/index.php/ijcrsee/article/view/108 
http://doi.org/10.15359/ree.25-1.11

http://www.una.ac.cr/educare

educare@una.ac.cr

Roig-Vila, R. (Ed.). (2016). EDUcación y TECnología. Propuestas desdela investigación y la innovación educativa. Octaedro. http://rua.ua.es/dspace/handle/10045/61788

Román, E. (2013). La dirección del trabajo independiente en la educación superior cubana. Reunión metodológica de apertura a la línea de trabajo de la Universidad de Sancti Spíritus para el curso 2012-2013", Sancti Spíritus. Material inédito.

Román-Cao, E. y Herrera-Rodríguez, J. I. (2010). Aprendizaje universitario centrado en el trabajo independiente. Educación y Educadores, 13(1), 91-106. https://doi.org/10.5294/ edu.2010.13.1.6

Román-Cao, E., Méndez-Hernández, O. y Pérez-García, Y. (2017). La educación a distancia centrada en el proceso de dirección del trabajo independiente. Pedagogía y Sociedad, 20(49), 205228. http://revistas.uniss.edu.cu/index.php/pedagogia-y-sociedad/article/view/547

Siemens, G. y Mateos, K. (2010). Systemic changes in higher education. In Education, 16(1), 3-18. https://ineducation.ca/ineducation/article/view/42/0

Silva Rodríguez, A., Mendoza Paredes, D. y Guarneros Reyes, E. (2009, setiembre). Sistema educativo mixto un modelo para la educación superior del futuro en México. Ponencia presentada en el $X$ Congreso Nacional de Investigación Educativa (pp. 1-11). Veracruz. http://www.comie.org.mx/congreso/memoriaelectronica/v10/pdf/area tematica 07/ ponencias/0875-F.pdf

Tabares Morales, V., Duque Méndez, N. D. y Ovalle Carranza, D. A. (2017). Modelo por capas para evaluación de la calidad de objetos de aprendizaje en repositorios. Revista Electrónica de Investigación Educativa, 19(3), 34-48. https://doi.org/10.24320/redie.2017.19.3.1128

Uribe Meneses, A. (2012). Características del aprendizaje autónomo de los estudiantes del programa de enfermería de la Universidad de Pamplona. Revista Ciencia y Cuidado, 9(1), 24-33. https://revistas.ufps.edu.co/index.php/cienciaycuidado/article/view/451

Vermunt, J. D. (1995). Process-oriented instruction in learning and thinking strategies. European Journal of Psychology of Education, 10(4), 325-349. https://www.jstor.org/stable/23420020

Weber, G. y Brusilovsky, P. (2001). ELM-ART: An adaptative versatile system for web-based instruction. International Journal of Artificial Intelligence in Education, 12, 351-384. http:// www.pitt.edu/ peterb/papers/JAIEDFinal.pdf 